\title{
Policy watch: revisions to the Animal Welfare Inspection Guide
}

\author{
B. Taylor Bennett \& Matthew R. Bailey
}

On June 23, 2017, the USDA APHIS

Animal Care (AC) released a Stakeholder's announcement pertaining to revisions to Chapters Two and Three of the Animal Welfare Inspection Guide (AWIG) (ID:APHISUSDA/Animal Welfare/ Animal-Care-Inspection-Guide) which addressed the issue of increasing consistency in four specific areas of the inspection process and the types of inspections carried out by the AC inspectors. The issue of increased consistency involved guidance on "teachable moments," identification of critical Non-Compliant Items (NCIs) to assure correct frequency of follow up inspections, guidance on the review of inspection and teachable moments reports, and documenting the requirements for documenting indoor/sheltered/outdoor housing facilities.

Here, we summarize several of the changes in AWIG of interest to those managing animal care and use programs (ACUPs) at registered research facilities. In the process of conducting our review, several questions arose which were addressed by representatives of the USDA who participated in NABR's July 18, 2017 webinar Q\&A with the USDA.

On pages 2-5 of AWIG is a list of all of the criteria that must be met in order for a teachable moment to be issued. Within that list, the phrase "Other Critical" appears. Critical NCIs include Direct NCIs while all other examples, found on pages $2-9$, are referred to as "Other Critical." The section entitled Recurring/Chronic NCI on pages 2-8 was moved from Chapter 3 with minor editorial changes. Inspection reports will not designate a Recurring/Chronic NCI, as is the case with Direct, Repeat and Critical NCIs.

National Association for Biomedical Research, Washington, DC, USA. Correspondence should be addressed to B.T.B. (btbdvm@yahoo.com).
"Critical" NCI Identified (a new section on pages 2-9) contains examples of issues classified as "Critical". Of particular interest to research facilities are issues that involve "a prior adverse event that had serious or severe animal welfare consequences to one or more animals, but poses no current risks to the animals." The majority of the critical citations reviewed appear to be based on information contained in the facility's records, specifically the IACUC minutes. Some of the examples of adverse events raised questions that we asked the USDA to clarify during the July 18 webinar. One involved a handling violation resulting in death or serious injury. This raised the question of whether a facility would be cited for incidents where a death or serious injury occurred as a result of another animal's actions. It would depend upon the history of the facility as it relates to monitoring animal interactions and making necessary changes when issues arise. The USDA recognizes that death and serious injury can result from another animal's actions with no previous history of aggression between animals, and where no other issues or citations exist, this would not be cited. Another example pertains to conducting procedures involving pain and distress without approval of the IACUC, which states "without appropriate response from the IACUC," implying if such activity was identified and handled correctly by the IACUC, there would be no citation. There are two scenarios where a facility could be cited: (1) if there was actual serious or severe animal welfare consequences, such as unrelieved pain or distress, regardless of the action taken by the IACUC; (2) where the IACUC had not taken any action regardless of whether the pain and distress was appropriately managed.

On pages 2-10 ("Direct" NCI Identified) is a statement we believed needed additional clarification involving "A prior adverse incident discovered during the inspection that had serious or severe animal welfare consequences and there are ongoing risks for the same serious or severe adverse effects at the time of the inspection should be marked as a Direct." An example of this would be equipment that resulted in a prior serious injury or death to an animal that was still malfunctioning with a high potential to cause additional serious injury or death in the future.

There are three areas addressing delivery of the inspection report. On pages 2-12 the requirement for an in-person exit interview has been strengthened and the language on pages $2-13$ requiring an explanation for the refusal of a facility representative to sign an inspection report has been expanded to include specifics regarding "who said what to whom, when, where, and how." On pages 3-20 (Completing the Inspection Report) is the requirement that inspection reports with Direct NCIs must be completed and delivered to the licensee/registrant immediately after the inspection.

The new section on the review of inspection and teachable moment reports on pages 2-22 requires the supervisor or their designee to review the inspection report in a timely manner and refers to checklists in Appendix A, pages A-28 and A-34, which should be reviewed by those managing ACUPs.

Finally, on pages 3-6, reference is made to an Environmental Enrichment Plan Checklist in Appendix A on page A-24. The checklist is not considered to be allinclusive, and is not retained in the facilities official AC file. Thus, it is not available under FOIA, but should be reviewed by those housing nonhuman primates.

While we consider the above sections to be some of the most relevant changes to AWIG, we encourage readers to do their own review of these chapters. 